\title{
Low-Level Laser Therapy and Vibration Therapy for the Treatment of Localized Adiposity and Fibrous Cellulite
}

\author{
Antonella Savoia $\cdot$ Simone Landi $\cdot$ \\ Fulvio Vannini $\cdot$ Alfonso Baldi
}

To view enhanced content go to www.dermtherapy-open.com

Received: April 2, 2013 / Published online: May 23, 2013

(c) The Author(s) 2013. This article is published with open access at Springerlink.com

\section{ABSTRACT}

Introduction: In recent years, there has been an upsurge in the application of low-level laser therapy in various medical diseases. Additionally, vibration therapy is a new and effective measure to prevent muscular atrophy and osteoporosis, along with some general health-related beneficial effects of exercise on skeletal muscles such as improvement of endothelial function and an increased enzyme capacity of energy metabolism. The aim of this study was to evaluate the application of a $635 \mathrm{~nm}$ and $0.040 \mathrm{~W}$ exit power per multiple diode laser in combination with vibration

A. Savoia $\cdot$ S. Landi $\cdot$ F. Vannini

Promoitalia Group S.p.A, Pozzuoli, Naples, Italy

A. Baldi $(\bowtie)$

Department of Environmental, Biological and

Pharmaceutical Sciences and Technologies, Second

University of Naples, Naples, Italy

e-mail: alfonsobaldi@tiscali.it therapy for the application of non-invasive reduction of circumference in patients with localized adiposity and cellulite.

Methods: The study enrolled men and women $(N=33)$ aged 18-64 years with localized adiposity or fibrous cellulite. The evaluation parameters were: photographic evaluation, perimetric evaluation, blood tests, ecographic evaluation, histological evaluation, and subjective and objective tests.

Results: The results produced were statistically analyzed and resulted in a significant reduction of fat thickness when compared to the measurement prior to the treatment $(P<0.0001)$. Moreover, subjective and objective tests, as well as ecographic and histological evaluations, confirmed the reduction of fat thickness.

Conclusion: In this study we have demonstrated the safety and efficacy of the combination between low-level laser therapy and vibration therapy for the resolution of localized adiposity and fibrous cellulite.

Keywords: Body contouring; Fibrous cellulite; Localized adiposity; Low-level laser therapy; Ultrasound; Vibration therapy 


\section{INTRODUCTION}

In recent years, there has been an upsurge in the application of low-level laser therapy (LLLT) across several neurologic, dental, ophthalmic, dermatologic disorders, and injuries [1, 2]. Furthermore, LLLT has been shown to be an efficacious adjunct therapy for numerous cosmetic procedures, including lipoplasty. Transmission electron microscopic images have demonstrated the formation of transitory pores in adipocyte cell membranes followed by the collapse of adipose cells subsequent to laser irradiation of $635 \mathrm{~nm}$ [3]. As such, LLLT at $635 \mathrm{~nm}$ has biochemical activity on adipocytes; indeed, LLLT stimulates the mitochondria in adipocytes to increase adenosine triphosphate (ATP) synthesis with subsequent up regulation of cyclic adenosine monophosphate (cAMP). The increase of cAMP stimulates cytoplasmic lipase which converts triglycerides into fatty acids and glycerol, which can pass through pores formed in the cell membrane [4]. On the other hand, vibration therapy is considered a new and effective strategy to prevent muscular atrophy and osteoporosis [5]. Vibration applied on skeletal muscles activates spindle receptors and so enlarges the drive to alpha-motoneurons via the monosynaptic reflex [6]. Furthermore, some general health-related beneficial effects of exercise on skeletal muscles such as improvement of endothelial function [7] and an increased enzyme capacity of energy metabolism have been described [8]. In particular, there are two specific effects of contracting skeletal muscle cells in their ability to transport glucose into these cells. First, a regularly performed training increases the content of glucose transporter protein GLUT-4 within the cells [9]. Second, a single bout of muscle contractions leads to a translocation of GLUT-4 to the sarcolemma membrane, which acutely enhances glucose transport capacity $[10,11]$.

Different clinical observations have established that treatment with LLLT at $635 \mathrm{~nm}$ is not sufficient for long-lasting and satisfactory results in reduction of circumference of adipose tissue. Often the results of the single LLLT are not durable. This is due, at least in part, to improper lifestyle and deficiency of physical activity. Therefore, taking into account this observation, we speculated that the combination of LLLT with vibration therapy, for its stimulation activity on muscle, on metabolism, and for its vascular and lymphatic drainage, could achieve more satisfactory results in reduction of localized adiposity and fibrous cellulite. The experimental objective of this work, therefore, was to evaluate the application of a $635 \mathrm{~nm}$ and $0.040 \mathrm{~W}$ exit power per multiple diode laser in combination with vibration therapy for the application of non-invasive reduction of circumference in patients with localized adiposity and cellulite.

\section{MATERIALS AND METHODS}

\section{Patient Population}

The study enrolled men and women $(N=33)$ aged 18-64 years with localized adiposity or fibrous cellulite. The patients were divided on the basis of the areas treated: abdomen and flank, buttocks, and thighs. Table 1 summarizes the characteristics of the patients. Before assessment and treatment, all patients received explanations about the procedure, after which they read and signed an informed consent form. All procedures followed were in accordance with the ethical standards of the responsible 


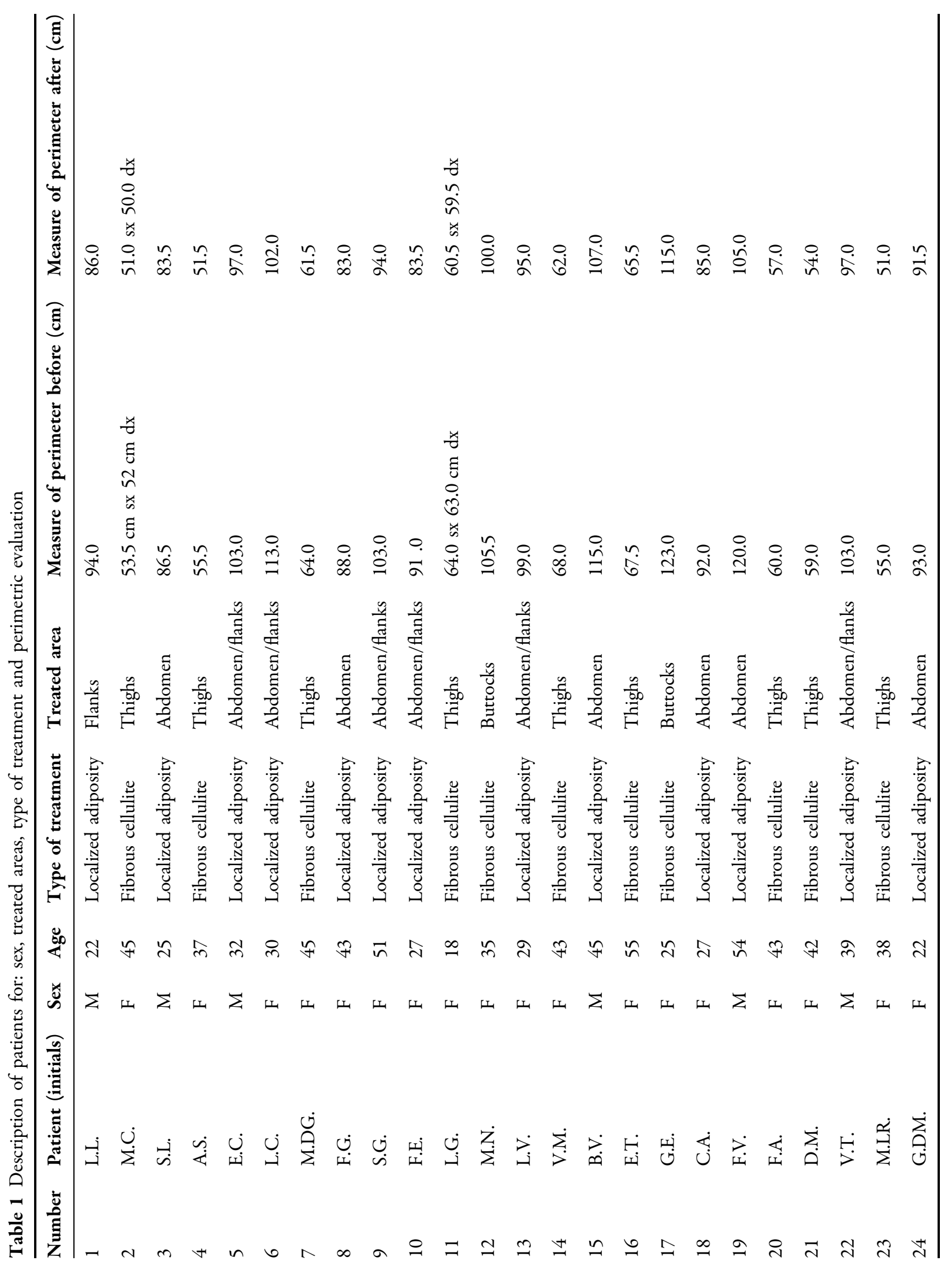




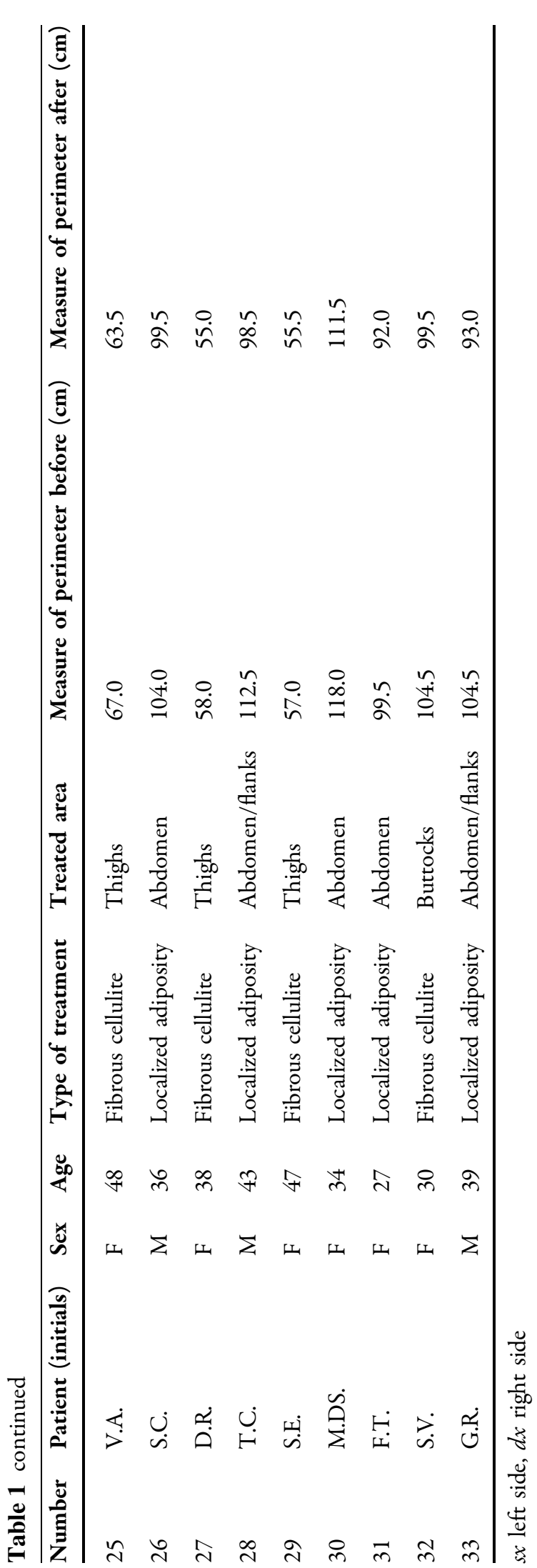

committee on human experimentation (institutional and national) and with the Helsinki Declaration of 1975, as revised in 2000 and 2008. Informed consent was obtained from all patients for being included in the study. Additional informed consent was obtained from all patients for whom identifying information is included in this article. The exclusion criteria were: diabetes mellitus, cardiovascular disease, prior cardiac surgery or implanted pacemaker, pregnancy, kidney stone, and mental disease. No patients underwent prior surgical or medical-aesthetic intervention for weight loss and reshaping. The subjects enrolled in this study have not changed their lifestyle, as determined through response to a patient questionnaire.

\section{Treatment Device}

The LLLT device consisted of eight independent probes, each with $6 / 12$ diode laser able to emit red light (635 wavelength) with $0.040 \mathrm{~W}$. The vibration therapy device consisted in a basculating platform with oscillating range from 4 to $25 \mathrm{~Hz}$. In detail, the apparatus used for this study was the Vibro Light $^{\mathrm{TM}}$ (Promoitalia Group S.p.A, Naples, Italy).

\section{Study Evaluation}

Each treatment was performed according to a default protocol. All the patients had a screening visit that included a physical examination and blood tests. The evaluation parameters were: (a) photographic evaluation before the first treatment and immediately after the last treatment; (b) physical evaluation consisting of measurement of the treatment zone before the first treatment, after every session of treatment, and 2, 4 weeks after the 
last session of treatment; (c) blood tests before and after the entire protocol. Furthermore, all patients completed a self-assessment test after the entire protocol about muscular tone and volume and were subjected to an objective test on muscular tone and volume after the entire protocol. Adverse events were recorded throughout the study.

\section{Study Design}

The treatment protocols were slightly different depending on the adiposity to be treated. In detail, for localized adiposity it consisted of one session per week for a total period of 6 weeks. Alternatively, for patients with fibrous cellulite, the protocol consisted of two sessions per week for a total of 4 weeks. At the beginning and at the end of the treatment programs, each subject's height and weight were recorded, and perimetric measures and photos of the area treated were taken. The hematic level has been verified with specific reference to the VLDL (fraction of cholesterol in which metabolization products of triglycerides contained in adipocytes pass), triglycerides, glutamate oxalate transaminase (GOT), and glutamate pyruvate transaminase (GPT) $24 \mathrm{~h}$ before the beginning of the first session, and at the end of the treatment.

Depending on the adiposity to be treated, patients were enrolled in two different protocols.

\section{Localized Adiposity}

Oscillating platform $6 \mathrm{~Hz}$ for $3 \mathrm{~min}$, LLLT $(635 \mathrm{~nm})$ for $10 \mathrm{~min}$, oscillating platform $9 \mathrm{~Hz}$ in combination with LLLT $(635 \mathrm{~nm})$ for $2 \mathrm{~min}$, oscillating platform $16 \mathrm{~Hz}$ in combination with LLLT $(635 \mathrm{~nm})$ for $5 \mathrm{~min}$, oscillating platform $7 \mathrm{~Hz}$ in combination with LLLT $(635 \mathrm{~nm})$ for 2 min. Total time 23 min.

\section{Fibrous Cellulite}

Oscillating platform $5 \mathrm{~Hz}$ for $3 \mathrm{~min}$, LLLT $(635 \mathrm{~nm})$ for $15 \mathrm{~min}$, oscillating platform $12 \mathrm{~Hz}$ in combination with LLLT $(635 \mathrm{~nm})$ for $5 \mathrm{~min}$, oscillating platform $5 \mathrm{~Hz}$ in combination with LLLT $(635 \mathrm{~nm})$ for $5 \mathrm{~min}$. Total time $28 \mathrm{~min}$.

The total time never exceeded $28 \mathrm{~min}$ in order to guarantee the highest comfort to the patients and to avoid an excessive body stress.

\section{Instrumental Evaluation}

Ultrasound surveys have been carried out before and after the treatment with the intent of evaluating the reduction of the hypodermic layer and the integrity of the surrounding structures.

\section{Histological Analysis}

A woman aged 45 needed to have surgical abdomino-plastic. After giving her informed

Table 2 Subjective evaluation: to show the level of satisfaction of patients about muscular tone and volume of the treated area

\begin{tabular}{llll}
\hline Score & $\begin{array}{l}\text { Results for } \\
\text { muscle tone } \\
\text { (number of } \\
\text { patients) }\end{array}$ & $\begin{array}{l}\text { Results for muscle } \\
\text { volume (number } \\
\text { of patients) }\end{array}$ \\
\hline $1 \quad$ Very & 20 & 22 \\
& satisfied & & \\
3 & $\begin{array}{c}\text { Somewhat } \\
\text { satisfied }\end{array}$ & 8 & 8 \\
3 & $\begin{array}{c}\text { Neither } \\
\text { satisfied }\end{array}$ & 4 & 1 \\
4 & $\begin{array}{c}\text { Not very } \\
\text { satisfied }\end{array}$ & 1 & 2 \\
5 & Not at all & 0 & 0 \\
\hline
\end{tabular}


consent, she agreed to have the skin of her abdomen treated with the study apparatus for the entire protocol before surgery. The surgery was carried out 7 days after the treatment under lumbar anesthesia and two representative fullthickness skin samples were taken at the same time, one at the site of the LLLT and Vibro Light application and one at the contra-lateral, symmetric untreated side to be examined by means of histopathology. Excised tissue samples were transferred in $4 \%(\mathrm{~m} / \mathrm{v})$ paraformaldehyde solution and paraffin embedded. Sections of $5 \mu \mathrm{m}$ were stained with haematoxylin-eosin, haematoxylin-van Gieson, and PAS-Alcian blue haematoxylin.

\section{Statistical Analysis}

Descriptive analysis was made using median values and 95\% confidence interval (CI). The differences in adiposity before and after treatment was performed using the Wilcoxon's test for non-parametric dependent continuous variables. The median reductions of adiposity according to the two methods were compared using the $U$ Mann-Whitney test for nonparametric independent variables. SPSS software (version 17.00, SPSS, Chicago) was used for statistical analysis. A $p$ value $<0.05$ was considered to indicate statistical significance.

\section{RESULTS}

Characteristics of the patients and measurement data are summarized in Table 1. Thirty-three patients were enrolled and all completed the study. Areas treated included the abdomen and/or flanks (18 patients), the thighs (12 patients), and the buttocks (3 patients). All patients were able to resume
Table 3 Objective evaluation: to show the change in appearance following the treatment evaluation about muscular tone and volume

\begin{tabular}{llll}
\hline Score & $\begin{array}{l}\text { Results for } \\
\text { muscle tone } \\
\text { (number of } \\
\text { patients) }\end{array}$ & $\begin{array}{l}\text { Results for muscle } \\
\text { volume (number of } \\
\text { patients) }\end{array}$ \\
\hline $1 \quad \begin{array}{c}\text { Improved } \\
\text { a lot }\end{array}$ & 18 & 21 \\
2 & $\begin{array}{c}\text { Improved } \\
\text { a little }\end{array}$ & 10 & 9 \\
3 & $\begin{array}{c}\text { No change } \\
\text { Worsened }\end{array}$ & 5 & 3 \\
4 & 0 & 0 \\
5 & W little & & 0 \\
\hline & Worsened & 0 &
\end{tabular}

normal activities upon completion of the session. The median fat thickness reduction at the end of the treatment was $6.83 \mathrm{~cm}$ for the abdomen and flanks, $3.42 \mathrm{~cm}$ for the thighs, and $6.16 \mathrm{~cm}$ for the buttocks. Final reduction of fat thickness was significant when compared to the measurement prior to the treatment $(Z=$ $-5.014, P<0.0001)$. The greatest reductions were observed in the abdomen and in the flanks, while the thighs showed the lowest reduction. There was no statistical difference in fat thickness reduction between men and women. Interestingly, patient weight remained constant over the treatment period in all patients, strongly suggesting that the fat thickness reduction was due to the treatment. Improvements in body contour were visibly appreciable in all patients at the end of the treatment, as supported by the data from the subjective evaluations, which showed always a clear satisfaction in all the patients treated (see Table 2). This was confirmed also by the objective test performed after entire protocol about muscular tone and volume (see Table 3). 

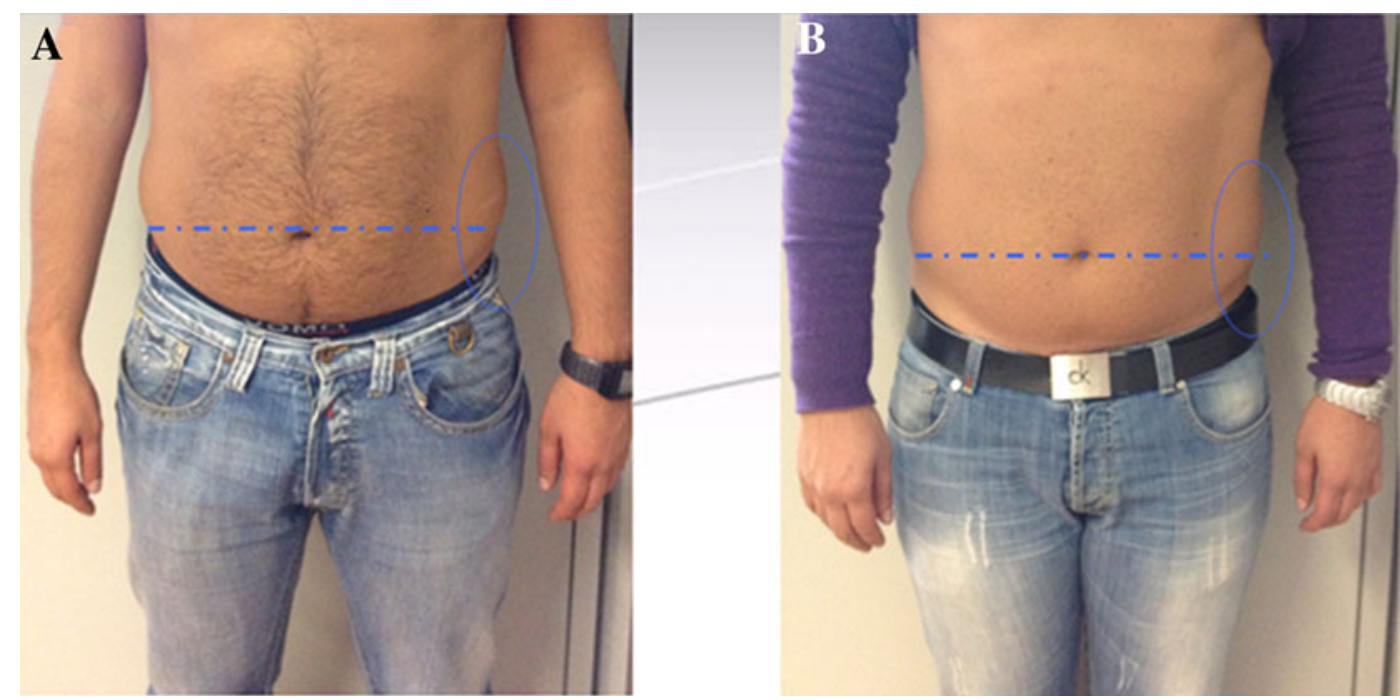

Fig. 1 Abdomen of a patient before treatment (a) and after treatment (b)
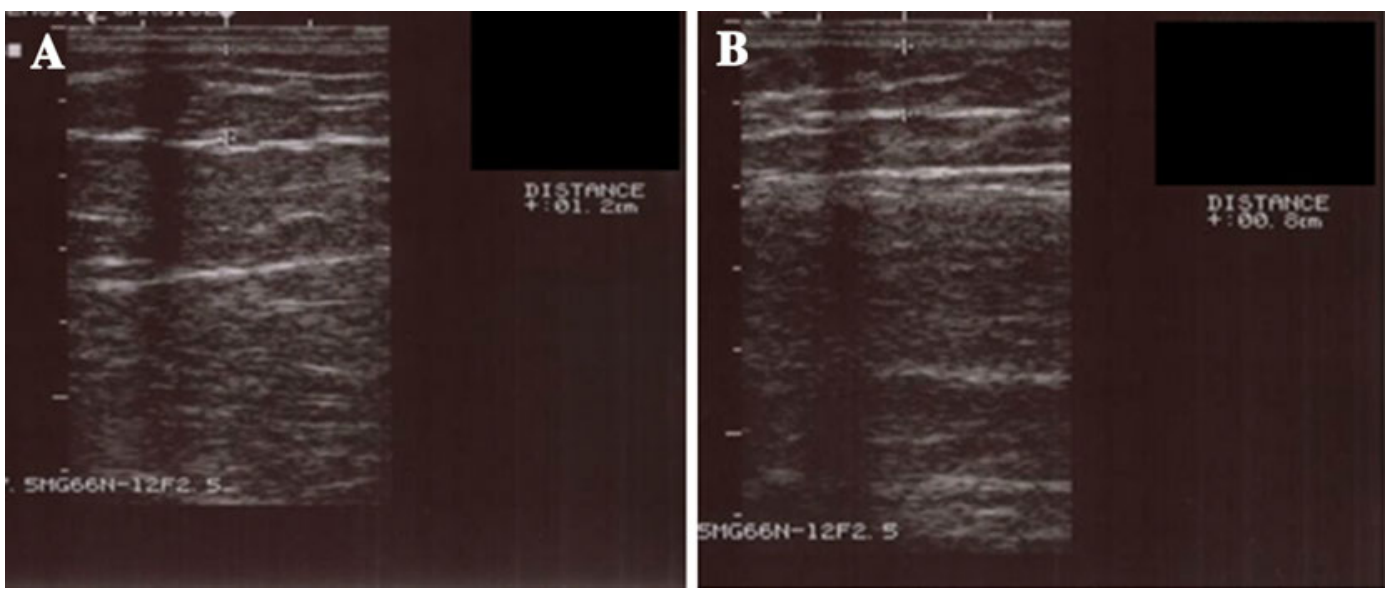

Fig. 2 Fat thickness assessment by ultrasound before treatment (a) and after treatment (b), shows thinning of the subcutaneous fat layer from 12.0 to $8.0 \mathrm{~mm}$ (reduction of $4 \mathrm{~mm}$ ). This patient was treated on the abdomen

In Fig. 1 an example of body contouring is depicted, while in Fig. 2 a representative ultrasound image pair, showing fat thickness before and after treatment of the abdomen is shown. Triglycerides and VLDL levels were mildly decreased after the treatments. Consistently, evaluation of the markers with hepatic functions (GOT, GPT, GT range) demonstrated no alterations in the hepatic functions. These data are summarized in Table 4. Finally, no severe adverse events were reported during and after the completion of the procedures; in particular no paresthesias, haematomas, ecchymoses, or oedema were noted or reported.

To further characterize the nature of the injury to the subcutaneous layer of treated skin, histopathological analysis of an area of the abdomen of a patient treated with LLLT and vibration therapy was performed and compared to another untreated area of the abdomen of the same patient. The histological analysis showed a clear reduction of fat thickness. Connective tissue, blood vessels and nerves had no 


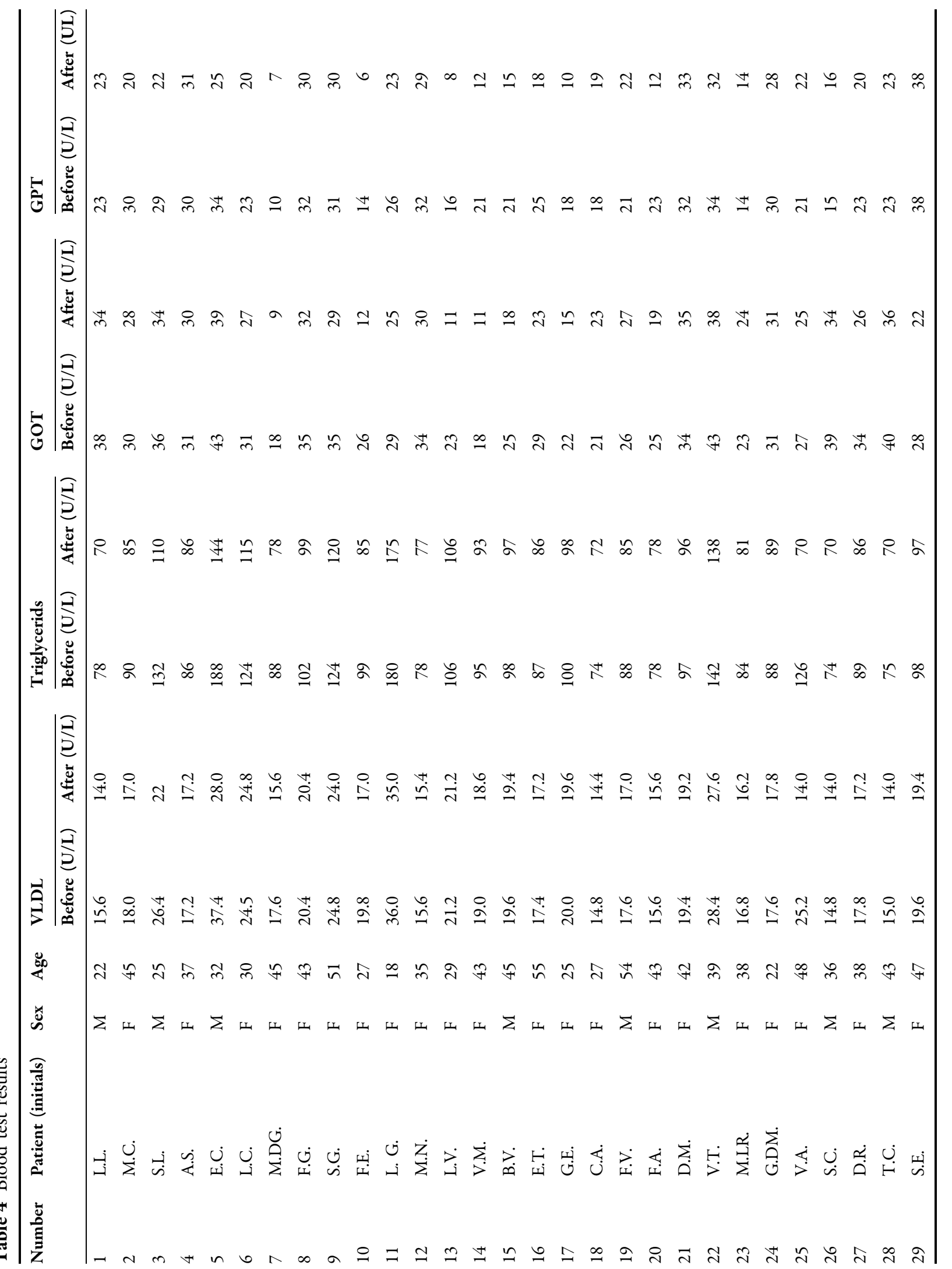




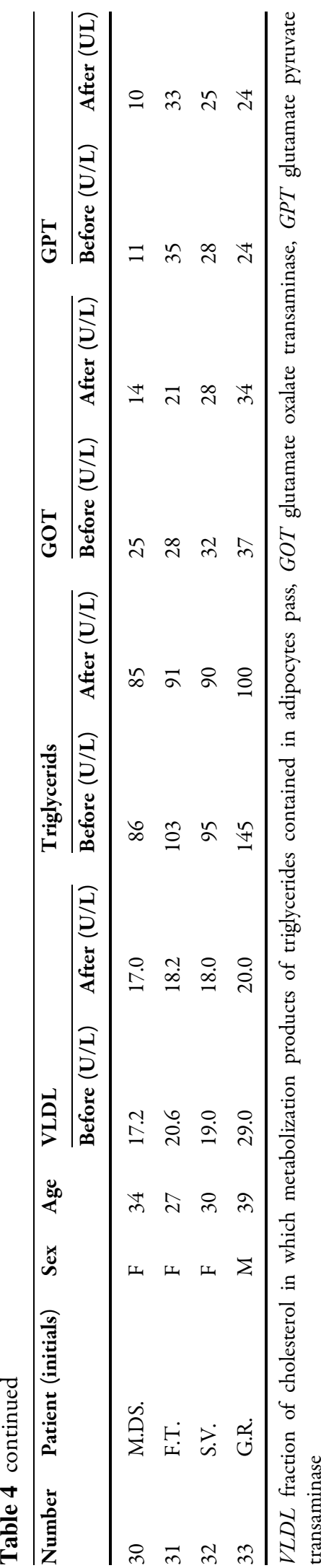

observable damage by haematoxylin and eosin and Van Gieson trichromic staining techniques. To note, no signs of tissue repair were visible, such signs of a response to an injury would be tissue-necrosis, extravasation of erythrocytes, the infiltration of neutrophils lymphocytes and macrophages and the subsequent scarformation. Logically, in the untreated skin specimen, all tissue components remained intact. In Fig. 3 the histological pattern of treated and untreated skins is depicted.

\section{DISCUSSION}

In recent years the mechanism of action of LLLT has been established. Neira et al. [3] examined the effect of LLLT at $635 \mathrm{~nm}$ with $10 \mathrm{~mW}$ intensity on human adipose tissue taken from lipectomy samples. Tissue samples were irradiated for $6 \mathrm{~min}$ and compared with nonirradiated samples. Utilizing scanning and transmission electron microscopy (SEM and TEM) more than 180 images were collected, and revealed that $99 \%$ of the cellular content including fat was released from the adipocyte, a phenomenon not observed within the control samples. Further, TEM images of the adipose tissue were taken at $60,000 \times$ and revealed the formation of transitory pore and complete deflation of adipocytes [3]. Over this effect, LLLT at $635 \mathrm{~nm}$ has biochemical activity on adipocytes. The biochemical effects of low-level laser energy laser on adipose tissue appears to be mediated through an effect on cytochrome C oxidase, a large mitochondrial membrane enzyme that facilitates the transfer of electrons from cytochrome $\mathrm{C}$ to oxygen. In detail, LLLT stimulates the mitochondria in adipocytes to increase adenosine triphosphate (ATP) synthesis with subsequent up regulation of cAMP. The increase of cAMP stimulates cytoplasmic lipase, 
which converts triglycerides into fatty acids and glycerol that can pass through pores formed in the cell membrane [4]. Following the application of low laser energy, fatty acids, glycerol, and triglycerides pass across the membrane through these transitory pores and into extracellular space resulting in complete adipocytes deflation.

Training using vibration platforms is a method of neuromuscular activation utilized for the development of strength and power. These vibrations transmit themselves through the body, causing a notable stimulation of sensorial receptors, principally the neuromuscular spindles. Recent studies have demonstrated that brief exposures to this type of treatment causes an acute increase in the muscular capacity of the subject, in both the upper [12] and lower [13] extremities. It has also been observed to produce an increase in the consumption of oxygen $\left(\mathrm{O}_{2}\right)$, of cardiac frequency (FC) and of the subjective perception of strength (RPE), similar to that produced with moderate resistance training [14].

In this study, we have tested the efficacy of a combination treatment with LLLT and vibration therapy for the treatment of localized adiposity and fibrous cellulite. Indeed, we have been able to demonstrate good results for the two pathological conditions, both at subjective and objective level.

Histopathological analysis has confirmed a significant reduction of the thickness of the cutaneous fat in the skin area treated with LLLT, with good preservation of the anatomical structures. In fact connective tissue, blood vessels and nerves had no observable damage by hematoxylin and eosin and Van Gieson trichromic staining techniques.

Ecographic evaluation confirmed the results of the histological exam, showing a measurable reduction of the fat thickness.

LLLT is a treatment able to increase the adipocyte metabolism with the consequent mobilization of the intracellular lipids. However, if not adequately metabolized, the lipids tend to re-set down invalidating the longterm effects of the treatment, especially in the absence of a correct lifestyle. Our data suggest that the combination of LLLT with vibration therapy is able to stimulate a metabolic and muscle stimulation, that are efficient in the consumption of the energy derived from the catabolism of the lipids mobilized by LLLT. Furthermore, the vibration therapy has also a strengthening effect on the skin.

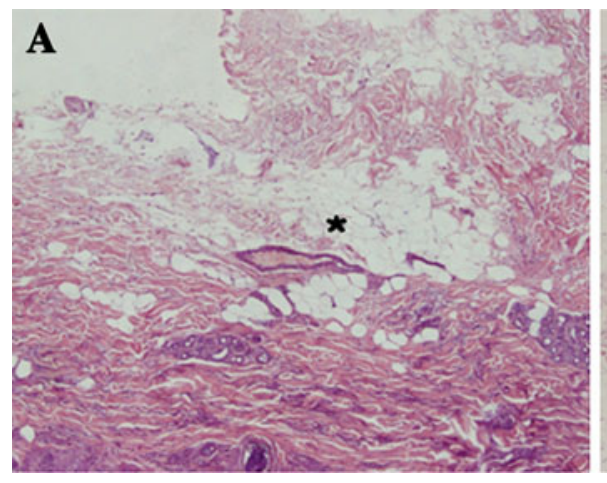

Fig. 3 Histopathology of skin and of subcutaneous fat tissue. One characteristic and representative slide taken from the central part of the skin-sample (a) and a corresponding slide (control) of the not treated skin-

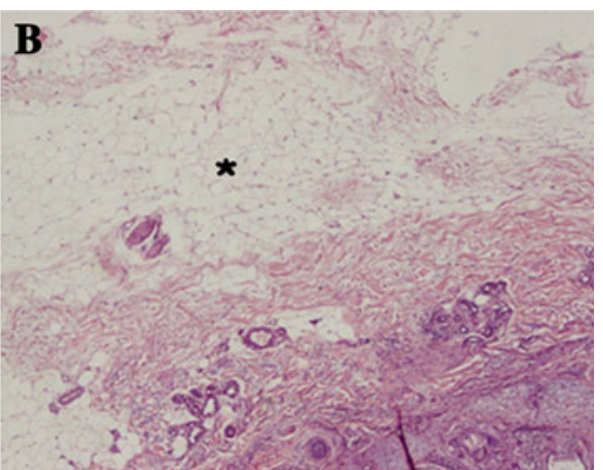

sample (b) are depicted. The reduction on fat thickness in the treated skin samples (indicated by asterisks) is observed (haematoxylin and eosin staining, original magnification $\times 20)$ 
The purpose of the study was to emphasize the effectiveness and safety of the combination of the two methods: LLLT and oscillating vibration technology. The sense of this combination arises from an attempt to improve the already satisfactory results of LLLT alone, as demonstrated in the literature, and to facilitate the use of mobilized free fatty acids following the adipocyte "depletion" induced by laser lipolysis. The oscillating vibration technology is not able to directly induce lipolysis, but provokes exclusively the basic processes involved in the pathogenetic mechanism of localized adiposity and cellulite [5]. Furthermore, it is able to stimulate the enzymatic processes involved in energy metabolism and to stimulate endothelial function and muscle due to stimulation of alpha motor neurons, resulting in an increase of muscle activity [6] and stimulation of $\mathrm{O}_{2}$ consumption, and of the basal metabolic rate [14]. Thus, in patients subjected to the combined therapy, such are those involved in this study, it was not essential to do physical exercise or follow a low-calorie/low-fat diet, in order to ensure the effectiveness and safety of the treatment.

Finally, blood tests have demonstrated the safety of the treatment. Indeed, all patients showed a slight decrease in VLDL and triglycerides. It is reasonable to hypothesize that the combination of LLLT with vibration therapy is able to induce an increase of the basal metabolism, which in turn could promote the consumption of the lipids mobilized from the fat tissue. Finally, we have never detected an increase in GOT and GPT levels, thus excluding any deleterious effect on the hepatic metabolism.

The relevant limitations of this study include: (1) metabolic data presented are sufficient to exclude toxic effects of the treatment, but are not enough to perfectly determine the biochemical events involved in the increase of the basal metabolism, that could be responsible, at least in part, for the effects on cellulite reduction; and (2) only one biopsy has been analyzed to investigate at the morphological effects of the treatment. This causes a limitation in the interpretation of the results.

In conclusion, the combination between LLLT and vibration therapy is an effective treatment for resolution of localized adiposity and fibrous cellulite. Moreover, the action of Vibro Light apparatus is safe, effective, and well tolerated as a procedure for body contouring. In particular, the study suggests that the combination treatment between LLLT and vibration therapy, using Vibro Light, could be an ideal non-invasive alternative to conventional liposuction for patients who require only small or moderate removal of adipose tissue or cellulite, and above all for those patients that refuse changes in their lifestyle through hyperproteic diet or appropriate physical activities. Considering the limitations of the study, further studies including a higher number of patients are required in order to better define the metabolic consequences of this treatment and the histological effects.

\section{ACKNOWLEDGMENTS}

Both the study and article processing charges were supported by Second University grants to Alfonso Baldi. Alfonso Baldi is the guarantor for this article, and takes responsibility for the integrity of the work as a whole.

Conflict of interest. Alfonso Baldi is a scientific adviser of Promoitalia Group S.p.A. 
Antonella Savoia, Simone Landi and Fulvio Vannini are consultants of Promoitalia Group S.p.A.

\section{Compliance with ethics guidelines. All} procedures followed were in accordance with the ethical standards of the responsible committee on human experimentation (institutional and national) and with the Helsinki Declaration of 1975, as revised in 2000 and 2008. Informed consent was obtained from all patients for being included in the study. Additional informed consent was obtained from all patients for whom identifying information is included in this article.

Open Access. This article is distributed under the terms of the Creative Commons Attribution Noncommercial License which permits any noncommercial use, distribution, and reproduction in any medium, provided the original author(s) and the source are credited.

\section{REFERENCES}

1. Stonecipher KG, Kezirian GM. Wavefrontoptimized versus wavefront guided LASIK for myopic astigmatism with the ALLEGRETTO WAVE: three-month result of a prospective FDA trial. J Refract Surg. 2008;24:S424-30.

2. Posten W, Wrone DA, Dover JS, Arndt KA, Silapunt S, Alam M. Low level laser therapy for wound healing: mechanism and efficacy. Dermatol Surg. 2005;31:334-40.

3. Neira R, Arroyave J, Ramirez $\mathrm{H}$, et al. Fat liquefaction: effect of low level laser energy on adipose tissue. Plast Reconstr Surg. 2002;110: 912-22.

4. Karu TI. Mitochondrial signaling in mammalian cells activated by red and near-IR radiation. Photochem Photobiol. 2008;84:1091-9.
5. Verschuren S, Roelants M, Delecluse C, et al. Effect of 6-month whole body vibration training on hip density, muscle strength and postural control in postmenopausal women: a randomized controlled pilot study. J Bone Miner Res. 2004;19:352-9.

6. Rotmuller C, Cafarelli E. Effect of vibration on antagonist muscle coactivation during progressive fatigue in humans. J Physiol (Lond). 1995;485: 857-64.

7. Green DJ, Maiorana AJ, Tschakovsky ME, et al. Relationship between changes in brachial artery flow-mediated dilation and basal release of nitric oxide in subject with type-2 diabetes. Am J Physiol Heart Circ Physiol. 2006;291:H1193-9.

8. Ostergard T, Andersen JL, Nyholm B, et al. Impact of exercise training on insulin sensitivity, physical fitness, and muscle oxidative capacity in first degree relatives of type 2 diabetic patient. Am J Physiol Endocrinol Metab. 2006;290:E998-1005.

9. Holten MK, Zacho M, Gaster M, et al. Strength training increases insulin-mediated glucose uptake, GLUT-4 content, and insulin signaling in skeletal muscle in patients with type 2 diabetes. Diabetes. 2004;53:294-305.

10. Gao J, Ren J, Gulve EA, Holloszy JO. Additive effect of contractions and insulin on GLUT-4 translocation into the sarcolemma. J Appl Physiol. 1994;77:1587-601.

11. Goodyear LJ, Hirshman MF, Horton ES. Exerciseinduced translocation of skeletal muscle glucose transporters. Am J Physiol Endocrinal Metab. 1991;f261:E795-9.

12. Bosco C, Cardinale $\mathrm{M}$, Tsarpela $\mathrm{O}$. Influence of vibration on mechanical power and electromyogram activity in human arm flexor muscles. Eur J Appl Physiol. 1999;79:306-11.

13. Torvinen S, Kannus P, Sievanen H, et al. Effect of four month vertical whole body vibration on performance and balance. Med Sci Sports Exerc. 2002;34:1523-8.

14. Rittweger J, Beller G, Felsenberg D. Acute physiological effects of exhaustive whole-body vibration exercise in man. Clinl Physiol. 2000;20: 134-42. 